\title{
Excitonic Crystal and Nanotechnology
}

\author{
Sergei Pyshkin* \\ Institute of Applied Physics, Academy of Sciences of Moldova, Republic of Moldova
}

Received: December 18, 2013; Accepted: April 04, 2014; Published: April 16, 2014

*Corresponding author: Sergei Pyshkin, Institute of Applied Physics, Academy of Sciences of Moldova, Republic of Moldova, Tel: $37322-796079$ 19; Fax: 37322- 7381 49; E-mail: spyshkin@phys.asm.md

\begin{abstract}
Since the time of original preparation of gallium phosphide crystals doped by nitrogen (GaP:N) by the author in the 1960s, followed by the introduction of the excitonic crystal concept in the 1970 s, the best methods of bulk, film and nanoparticle crystal growth were elaborated. The results of semi centennial evolution of GaP:N properties are compiled here and in the references to this paper. Novel and useful properties of perfect GaP including an expected similarity in behavior between nanoparticles and perfect bulk crystals as well as their very bright and broadband luminescence at room temperature were observed. These results provide a new approach to selection and preparation of perfect materials for optoelectronics and a unique opportunity to realize a new form of solid-state host - the excitonic crystal. The excitonic crystal (filled by non-equilibrium electron-hole pairs $\mathrm{N}$ super lattice with the identity period equal to $10 \mathrm{~nm}$ - the Bohr dimension of bound exciton) as high intensity light source with expected low threshold for generation of non-linear optical effects, as well as GaP nanoparticles and bulk top quality crystals will be used in the new generation of optoelectronic devices of solid-state host - the excitonic crystal.
\end{abstract}

Keywords: Semi centennial evolution of GaP:N properties; Longterm ordering; Excitonic crystal

\section{Excitonic Crystal and its Importance in Optoelectronics}

The role and application of bound excitons in nanoscience and technology are discussed in this chapter. Bound excitons are well studied in semiconductors, especially in gallium phosphide doped by nitrogen (GaP:N). Doping of GaP with $\mathrm{N}$ leads to isoelectronic substitution of the host $\mathrm{P}$ atoms by $\mathrm{N}$ in its crystal lattice and to creation of the electron trap with a giant capture cross-section. Therefore, any non-equilibrium electron in the vicinity of the trap will be captured by $\mathrm{N}$ atom, attracting a nonequilibrium hole by Coulomb interaction and creating the bound exciton - short-lived nanoparticle with the dimension of the order of $10 \mathrm{~nm}$ (it is the Bohr diameter of bound exciton in GaP:N). Note, that none of nanotechnology methods are used in creation or selection of dimensions of these nanoparticles - only natural forces of electron-hole interaction and electron capture by the traps are necessary for creation of these nanoparticles. As the result we get something like neutral short-lived atom analogue a particle consisting of heavy negatively charged nucleus ( $\mathrm{N}$ atom with captured electron) and hole. So called "zero vibrations" do not destroy possible solid phase of bound excitons having these heavy nuclei that gives an opportunity to reach their crystal state - short-lived excitonic crystal.

Thus using bound excitons as short-lived analogues of atoms and sticking to some specific rules, including the necessity to build in the GaP:N single crystal the excitonic super lattice with the identity period equal to the bound exciton Bohr dimension, we get a unique opportunity to create a new solid state media - consisting from short-lived nanoparticles excitonic crystal, obviously, with very useful and interesting properties for application in optoelectronics, nanoscience and technology. The following will discuss methods of preparation and possible application of GaP excitonic crystals and nanocrystals in optoelectronics.

\section{Preparation and Properties of GaP with Ordered Position of N Impurities}

Crystals that are grown under conventional laboratory conditions naturally contain a varied assortment of defects such as displaced host and impurity atoms, vacancies, dislocations, and impurity clusters. These defects result from relatively rapid growth conditions and inevitably lead to the deterioration of optical and mechanical properties of the crystal. For instance, defects in freshly-prepared GaP:N single crystals completely suppress their luminescence at room temperature, which is very bright in the same, but aged perfect crystal.

With the lapse of time driving forces such as impurity diffusion, strain relaxation, and thermodynamic minimization of the free energy associated with properly directed chemical bonds can result in an ordered distribution of impurity and host atoms. Evaluation of the characteristic time of such reordering, based on the known Ising model, suggests that the time for the substitution reaction associated with $\mathrm{N}$ diffusion along $\mathrm{P}$ sites in GaP:N is about 15 years at room temperature [1]. Accordingly, observations of the luminescence from GaP: $\mathrm{N}$ crystals made at 10-15 year intervals under similar experimental conditions were used successfully to track such structural evolution [2-15]. In this paper, as well as in the works published in 2012-2013 [14-16] we report the cumulative effects of over 50 years of lattice ordering on properties of GaP single crystals, providing clear evidence for enhanced optoelectronic properties through temporal dynamics over long time periods. 
Figure 1 provides a comparison of the evolution in luminescence spectra at low temperatures ( $80 \mathrm{~K}$ and below) from GaP:N over a period of 25 years (original measurement in about 1963). Over this period, zero-phonon line A of single $\mathrm{N}$ impurity-bound excitons and their phonon replicas are narrower in their line-widths when compared to the freshly prepared single crystals. Further, as expected, zero phonon line and replica in samples aged at room temperature for 25 years shift spectral position depending upon concentration of $\mathrm{N}$ impurities (Figure 1b, spectra 1-3) according to[17], while the same freshly prepared crystals exhibited broader luminescence line-widths with increasing nitrogen content (Figure $1 \mathrm{~b}$, spectrum 4). These, along with other half-centennial findings, including modifications to luminescence kinetics, spontaneous Raman scattering, x-ray diffraction, absorption spectra, micro-hardness and density of dislocations, that are reported elsewhere [2-16] strongly suggest that close-to-ideal GaP:N crystals are formed over time through the equally-spaced disposition of $\mathrm{N}$ impurities from their chaotic distribution in the same freshly prepared crystals.

As first noted in Ref [11], these results suggest a new type of crystal lattice in which the host atoms occupy their proper (equilibrium) positions in the crystal, while the $\mathrm{N}$ impurities, periodically substituted into the lattice portion it into short chains of equal length. According to the data obtained from Raman light scattering $[9,13]$ host atoms of this new lattice develop harmonic vibrations, and high degree of lattice perfection leads to an abrupt decrease in the non-radiative recombination and an increase of efficiency and spectral range of luminescence. Additionally, stimulated emission of light in these temporally-ordered crystals (Figure 2b) is observed. As also shown in Ref [11] the GaP:N crystals aged for at least 40 years possess no discrete impurity level for $\mathrm{N}$-bound excitons in the forbidden gap. They also demonstrated a uniform luminescence from a broad excitonic band instead of the narrow zero-phonon line and its phonon replica as observed from the less-aged 25 year-old crystals.

Thus, long-term ordered GaP:N crystals demonstrate uniform

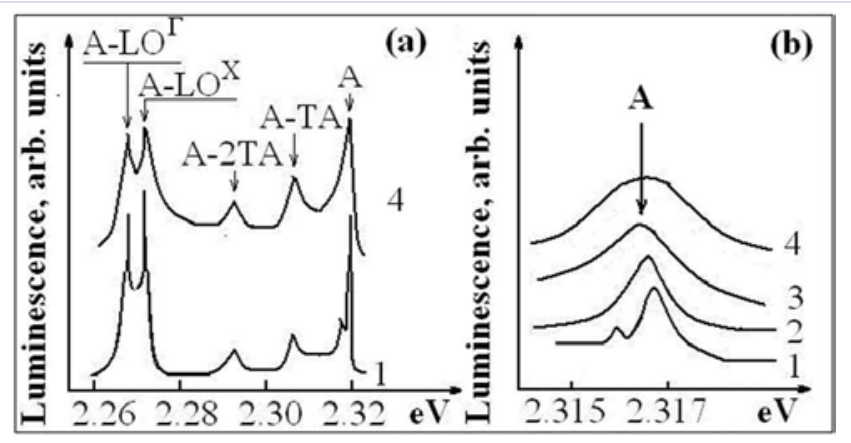

Figure 1: Evolution of the GaP:N luminescence with time and nitrogen concentration at the temperature of $15 \mathrm{~K}$. (a) Zero-phonon line of the bound exciton $A$ and its transversal acoustic (TA) and longitudinal optic (LO) phonon replica in as-prepared (4) and 25-year long-term ordered (1) crystals. (b) Zero-phonon line A as a function of nitrogen (N) concentration. 1-3: 25-year-old crystals. 4: as-prepared. Curves 1 through 4 represent samples with nitrogen concentrations of 1017, 1018, 1019, and $1018 \mathrm{~cm}-3$, respectively. luminescence from a broad excitonic band instead of the narrow zero-phonon line and its phonon replica in disordered and partly ordered (25-year-old) crystals. This is due to the fact that ordered crystals have no discrete impurity level in the forbidden gap. To the best of our knowledge, such transformation of a discrete level within the forbidden gap into an excitonic band (Figure. $2 \mathrm{a}, \mathrm{b}$ ) is observed for the first time. In this case, the impurity atoms regularly occupy the host lattice sites and affect the band structure of the crystals, which is now a dilute solid solution of GaP-GaN with regular disposition of $\mathrm{N}$ atoms rather than GaP doped by occasionally located $\mathrm{N}$ atoms. Note that the increase of luminescence excitation in the case of partly ordered GaP:N (Figure 2a, dotted line) leads to a broad luminescence band as a result of bound exciton interaction[18], while in the case of perfectly ordered crystals (Figure $2 b$ ) one can see an abrupt narrowing of the luminescence band, probably, due to stimulated emission in defect-free crystals. Earlier, in freshly prepared crystals, we observed a clear stimulated emission from a GaP:N resonator at $80 \mathrm{~K}$ [3], as well as the so called super luminescence from the GaP single crystals having natural faceting. Presently, our ordered crystals have a bright luminescence at room temperature that implies their perfection and very low light losses. In our studies $[11,13]$ we demonstrate that the stimulated emission is also developed even at room temperature by direct electron-hole recombination of an electron at the bottom of the conduction band with a hole at the top of the valence band and the LO phonon absorption.

If the nitrogen content is selected in such a way that the length of the chain is about $10 \mathrm{~nm}$, corresponding to the Bohr diameter of an exciton bound to $\mathrm{N}$, then the relevant concentration of non-equilibrium electron-hole pairs captured by $\mathrm{N}$ traps creates an excitonic crystal. A schematic model for this novel excitonic crystal is shown in Figure 2c. In the presence of heavier nuclei (N atom + captured electron) decay of the solid excitonic phase due to so-called "zero vibrations" becomes impossible and the phase itself will serve as an efficient accumulator of light and a new prospective optic media with low nonlinear thresholds.

Figure 3 compares the luminescence spectra of our longterm (up to 50 years) ordered GaP single crystals (spectrum 1) to that from high quality GaP nanoparticles and their GaP nanoparticles/polymers nanocomposites [15]. Nanocrystals stored as dry powder demonstrate rather broad luminescent band with maximum at $2.8 \mathrm{eV}$ (Figure 3, spectrum 2), while the nanocrystals, all of about $10 \mathrm{~nm}$ in size, thoroughly separated and distributed in a suspension, which prevents their coagulation, mechanical and optical interaction, exhibit a bright narrow-band luminescence with maximum at $3.2 \mathrm{eV}$, approximately $1 \mathrm{eV}$ above the position of the absorption edge in $\mathrm{GaP}$ at $300^{\circ} \mathrm{K}$ (Figure3, spectrum 3).

The thoroughly washed, ultrasonicated and dried nanopowders as well as their specially prepared suspensions have been used for fabrication of blue light emissive GaP nanocomposites on the base of some optically and mechanically compatible with GaP polymers $[19,15]$. 
We explain the broadening of the luminescence band and the shift of its maximum to low photon energies in luminescence of the nanocomposite based on the GaP powder (Figure 3, spectrum 2)by presence in the powder of the nanoparticles with different dimensions between 10-100 $\mathrm{nm}$. Meanwhile, the nanocomposites on the base of the suspensions containing only approximately 10 $\mathrm{nm}$ nanoparticles exhibit bright luminescence with maximum at $3.2 \mathrm{eV}$. This is due to high transparency of $10 \mathrm{~nm}$ nanoparticles for these high energy emitted photons and pronounced quantum confinement effect.

In order to explain this interesting phenomenon, we postulate that the nanocrystals, much like the ideal long-term ordered bulk GaP single crystals, exhibit this huge increase in blue-shifted luminescence due to: (a) negligibly small influence of defects and

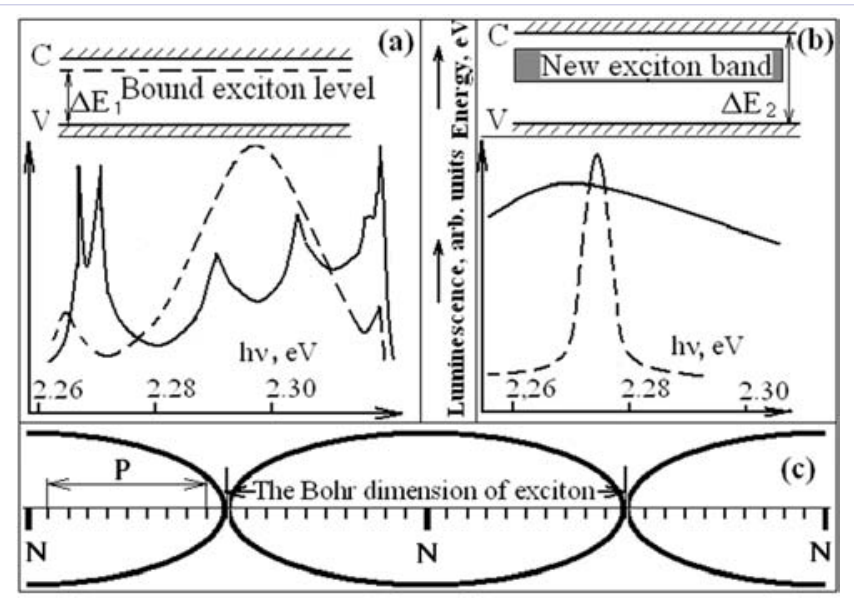

Figure 2: Luminescent spectra and schematic representation of the forbidden gaps $(\Delta \mathrm{E} 1, \Delta \mathrm{E} 2)$ in the nitrogen-doped GaP aged for (a) 25 years and (b) 40 years. The dotted lines correspond to highly optically excited crystals. $\mathrm{C}$ and $\mathrm{V}$ represent the positions of the bottom of the conductance and the top of valence bands, respectively. (c) A schematic representation of the excitonic crystal in highly optically excited long-term ordered nitrogen-doped GaP:N. N substitutes the host P atoms with the period equal to the Bohr exciton diameter.

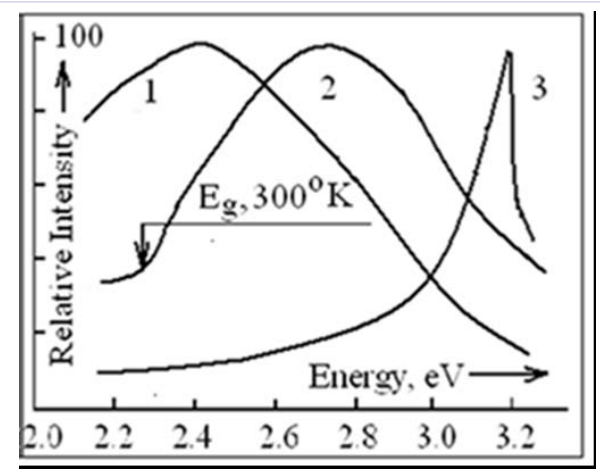

Figure 3: Luminescence of perfect bulk GaP single crystals (1) in comparison with the luminescence of GaP nanoparticles and GaP/polymers nanocomposites $(2,3)$.Nanoparticles were prepared from white $\mathrm{P}$ by mild aqueous or colloidal synthesis at decreased temperature and stored as the dry powder (spectrum 2) or suspension in a liquid (spectrum 3). Details: [Pyshkin and Ballato, 2011, 2013]. non-radiative recombination of electron-hole pairs and very high efficiency of their radiative annihilation, (b) high perfection of nanocrystal lattice, and (d) high transparency of nanocrystals due to their small dimensions for the light emitted from high points of the GaP Brillouin zones, for instance, in the direct transitions $\Gamma_{1}{ }^{\mathrm{c}}$ - $\Gamma_{15}$ v between the conductive and valence bands with the photon energy at $300^{\circ} \mathrm{K}$ equal to $2.8 \mathrm{eV}$ [20] and (e) high efficiency of this so called "hot" luminescence.

Our first attempts to prepare GaP nanoparticles [21] yielded room temperature luminescence with maximum shifted only to $2.4 \mathrm{eV}$ in comparison with the new maximum at $3.2 \mathrm{eV}$. It confirms significant achievements in technology of GaP nanoparticles and $\mathrm{GaP} /$ polymers nanocomposites. On the base of these improved technologies for preparation of $\mathrm{GaP}$ nanoparticles and GaP/ polymer nanocomposites we can change the main parameters of luminescence within broad limits. And we can expect to create a framework for novel light emissive device structures using dramatic $1 \mathrm{eV}$ expansion of GaP luminescence to UV region.

Note, that luminescence from freshly grown GaP crystals has always been observed at low temperatures and at photon energies less than the forbidden gap $(\sim 2.3 \mathrm{eV})$. However, as shown here, after 50 years, bright luminescence of the long-term ordered bulk GaP crystals clearly is detected in the region from $2.0 \mathrm{eV}$ to $3.2 \mathrm{eV}$ at room temperature (see Figure 3, spectrum 1). It is worth noting further [22] that the absorption coefficient near $3.0 \mathrm{eV}$ exceeds $10^{5} \mathrm{~cm}^{-1}$. Obviously, decreasing the thickness of the crystal will increase the contribution from high energy photons and shift the spectral maximum into the ultraviolet. This significant ability to achieve a dramatic $1 \mathrm{eV}$ expansion of luminescence into the ultraviolet at room temperature is likely due to small concentration of defects, low contribution of non-radiative electron-hole recombination, considerable improvement in the crystal lattice perfection over time - all of which contribute to an increased transparency and higher probability for the emission of higher energy photons.

It is worth noting that known quantum confinement effect in GaP nanoparticles yields only a few tenths of eV contribution to the spectral blue-shift and therefore cannot be the main cause of this significant $1 \mathrm{eV}$ shift unto the UV.

Thus, well-aged GaP bulk crystals [16] as well as high quality GaP nanoparticles [23-26] have no difference in their luminescence behavior, interesting for application in different fields.

\section{Conclusion}

Since the time of original preparation of gallium phosphide doped by nitrogen crystals (GaP:N) by the author in the 1960s, followed by the introduction of the excitonic crystal concept in the 1970s, the best methods of bulk, film and nanoparticle crystal growth were elaborated. The results of semi centennial evolution of GaP:N properties are compiled here and in the references to this paper. Novel and useful properties of GaP including an expected similarity in behavior between nanoparticles and perfect bulk crystals, as well as very bright and broadband luminescence at 
room temperature, are observed. These results provide a new approach to selection and preparation of perfect materials for optoelectronics [16] and a unique opportunity to realize a new form of solid-state host - the excitonic crystal. In spite of the fact that the time necessary for natural long-term ordering (years) does not lead to optimism, the collected experience and results confirm expedience of the efforts directed to formation in GaP of the $\mathrm{N}$ impurity superlattice having the identity period equal to the bound exciton dimension. As noted in Ref [16] the process of preparation of top quality material for industrial electronics can be organized if the freshly grown crystals will be kept for years in a special storage and only old crystals with the necessary properties will be annually retrieved for device fabrication while new portions of fresh crystals will be placed for long-term ordering. Except natural aging of the relevant crystals for years, preparation of the $\mathrm{N}$ super lattice for excitonic crystal can be also realized by known methods of growth of multi-layer films, in particular, by molecular beam and laser assisted epitaxy [27].

The excitonic crystal, created by the long-term ordering or by the noted above methods of growth of multi-layer films, as well as the bulk top quality GaP crystals with the unique optic properties, obtained by the long-term ordering process of freshly prepared crystals, will be used in new generation of optoelectronic devices, sometimes instead of nanoparticles and a lot of other materials. In particular, keeping in mind the low energy of the bound exciton creation, one can expect a low threshold for generation of non-linear optical effects in the excitonic crystal and a good opportunity to create new and very efficient optoelectronic devices.

Note, semiconductor nanoparticles were introduced into materials science and engineering mainly in order to avoid limitations inherent to freshly grown semiconductors with a lot of different defects. However, it was shown in [19] that this reason becomes unessential if, when justified, perfect longterm ordered semiconductor crystals are applied in electronics. Independently on their dimensions they demonstrate very interesting for application properties. Therefore, using the longterm ordered, perfect GaP crystals or similar on behavior and properties material in electronic industry instead of elaboration of very expensive and labor-consuming technologies for diverse materials and their nanoparticles with limited for application spectral region and other parameters, we get a big commercial advantage from their fabrication and application [for more details please see the paper [16]. All the results noted above and included in summary reviews $[15,16,19]$ may sufficiently change the approach to selection of materials necessary for electronics, to make cheaper and to simplify technology of preparation of the selected materials and device structures based on them.

\section{Acknowledgment}

The author is glad to note that the broad discussion and dissemination of our results stimulate successful collaboration with reliable partners from USA, Russia, Italy, Romania, France and other countries. The author is very grateful to the US Department of State, Institute of International Exchange, Washington, DC, The US Air Force Office for Scientific Research, the US Office of Naval Research Global, Civilian R\&D Foundation, Arlington, VA, to his colleagues and co-authors from Clemson University, SC, University of Central Florida, FL, Institute of solid state electronics, CNR, Rome, Italy, University of the studies, Cagliari, Italy, Lomonosov Moscow State University, Ioffe Physico-Technical Institute and State Polytechnical University, St. Petersburg, Ac. Scie. Institute of General Physics, Moscow, Russia, and Institute of Applied Physics Academy of Sciences of Moldova for support and attention to this protracted (1963-present time) research.

\section{References}

1. Pyshkin S, Budyanu VA, Damaskin IA, Zenchenko Val P, Radautsan SI, et al. (1990) Processes of Long-Lasting Ordering in Crystals with a Partly Inverse Spinel Structure. J Soviet Physics Doklady 35(4): 301304.

2. Pyshkin SL (1967) Preparation and Properties of Gallium Phosphide. Ph.D. thesis, State University of Moldova, Kishinev.

3. Pyshkin SL (1975) Stimulated emission in gallium phosphide. J Soviet Physics Doklady 19: 845-846.

4. Pyshkin S (1975) Luminescence of GaP:N:Sm Crystals. J Soviet Physics of Semiconductors 8: 912-913.

5. Pyshkin SL (1978) Photoconductivity and luminescence of highly optically excited semiconductors. Dr Sc thesis, Lomonosov Moscow State University

6. Pyshkin S (1969) Influence of Impurities and Crystallization Conditions on Growth of Platelet GaP Crystals (presented at the 7th Int. Crystallography Congress, Moscow, 1966). J Growth of Crystals ed Sheftal NN New York 8: 68-72.

7. Pyshkin SL, Zifudin L (1974) Excitons in highly optically excited gallium phosphide. J Luminescence 9: 302-308.

8. Pyshkin S, Anedda A, Congiu F, Mura A (1993) Luminescence of the GaP:N ordered System. J Pure and Applied Optics 2: 499-502.

9. Pyshkin S, Ballato J, Chumanov G (2007) Raman light scattering from long-term ordered GaP single crystals. Journal of Optics A Pure and Applied Optics 9: 33-36.

10. Pyshkin SL, Ballato J, Bass M, Turri G (2008) Luminescence of longterm ordered pure and doped gallium phosphide (TMS Annual Meeting, invited talk at the Symposium: Advances in Semiconductor, Electro Optic and Radio Frequency Materials, March 9-13, New Orleans, LA). J Electronic Materials 37(4): 388-395.

11. Pyshkin SL, Ballato J, Bass M, Turri G (2009) Evolution of luminescence from doped gallium phosphide over 40 Years. J Electronic Materials 38(5): 640-646.

12. Pyshkin SL, Ballato J, Bass M, Chumanov G (2009) Time-dependent evolution of crystal lattice, defects and impurities in $\mathrm{CdIn}_{2} \mathrm{~S}_{4}$ and GaP. J Phys Status Sol (6): 1112-1115.

13. Pyshkin S, Ballato J (2010) Evolution of optical and mechanical properties of semiconductors over 40 Years. J Electronic Materials 39(6): 635-641.

14. Pyshkin S, John B (2012) Dramatic Expansion of Luminescence Region in GaP/Polymer Nanocomposites. Supplemental Proceedings of the 2012 TMS Annual Meetings (Orlando, FL, USA, March 11-15, 2012) 1: 353-359. 
15. Pyshkin S, Ballato J (2013) "Advanced Light Emissive Device Structures", Chapter 1 in the book "Optoelectronics - Advanced Materials and Devices", Editors: Sergei L. Pyshkin and John Ballato, InTech Open Access Publisher: 1-24.

16. Pyshkin SL (2013) Gallium Phosphide - New Prospect for Optoelectronics. J Advances in Optoelectronic Materials 1(4): 59-66.

17. Allen WJ (1968) Energy levels of nitrogen-nitrogen pairs in gallium Phosphide. Journal of Physics C: Solid State Physics 1: 1136-1138.

18. Pyshkin, Sergei and Alberto Anedda, (1998). Time-Dependent Behaviour of Antistructural Defects and Impurities in Cd-In-S and GaP. Institute of Physics Conference Series, Ternary and Multinary Compounds 152: 785-789.

19. Pyshkin SL, Ballato J (2011a) Long-Term Convergence of Bulk- and Nano-Crystal Properties. Chapter 19 in "Optoelectronics - Materials and Technics". InTech Open Access Publisher.

20.Zallen R, Paul W (1964) Band Structure of Gallium Phosphide from Optical Experiments at High Pressure". Phys Rev 134: A1628-A1641.

21. Pyshkin, S, John B (2005) Advanced Light Emissive Composite Materials for Integrated Optics. Symposium: The Physics and Materials Challenges for Integrated Optics - A Step in the Future for Photonic Devices, Proc. of the 2005 MS\&T Conference Pittsburgh: 3-13.
22. Dean P J, Kaminsky G, Zetterstrom RB (1967) Intrinsic optical absorption of Gallium Phosphide between 2.33 and $3.12 \mathrm{eV}$. J Appl Phys 38: 3551-3556.

23. Pyshkin S, Ballato J, Chumanov G, Tsyntsaru N, Rusu E (2010) Preparation and characterization of nanocrystalline GaP for advanced light emissive device structures. In: The 2010 NSTI-Nanotech Conference (Anaheim, CA) Proceedings 1: 522-525.

24. Pyshkin SL, Ballato J, Belevschii S, Rusu E, Racu A, et al. (2011) Synthesis and characterization of GaP nanoparticles for light emissive devices. In: The 2011 NSTI-Nanotech Conference, Boston, MA, Proceedings: 327-330.

25. Pyshkin S, Ballato J (2011b) Long-Term Convergence of Bulk- and Nanocrystal Properties (The 2010 Materials Science \& Technology 2010 Conference, Symposium: Advances and Applications in Electroceramics, October 17-21, Houston, Texas) Ceramic Transactions 226: 77-90.

26. Pyshkin SL, Ballato J, Luzinov I, Zdyrko B (2011) Fabrication and Characterization of GaP/Polymer Nanocomposites for Advanced Light Emissive Device Structures. Journal of Nanoparticle Research 13: 5565-5570.

27. Pyshkin SL, Fedoseev SA, Lagomarsino S, Giannini C (1992) Preparation and Structural Properties of Some III-V Semiconductor Films Grown on (100) Oriented Si Substrates. Appl Surf Scie 56-58: $39-43$. 